\title{
Fallopian Tube Perforation, CTCAE 5.0
}

National Cancer Institute

\section{Source}

National Cancer Institute. Fallopian Tube Perforation, CT CAE 5.0. NCI Thesaurus. Code C146714.

A disorder characterized by a rupture of the fallopian tube wall. 